\title{
Semi-Automatic 4D Fuzzy Connectedness Segmentation of Heart Ventricles in Cine MRI
}

\author{
Markus Hüllebrand ${ }^{1}$, Anja Hennemuth ${ }^{1}$, \\ Daniel Messroghli ${ }^{2}$,Titus Kühne ${ }^{2}$,Ola Friman ${ }^{1}$ \\ ${ }^{1}$ Fraunhofer MEVIS - Institute for Medical Image Computing, Bremen, Germany \\ ${ }^{2}$ German Heart Institute Berlin, Berlin, Germany \\ markus.huellebrand@mevis.fraunhofer.de
}

\begin{abstract}
The volumes of the left and right cardiac ventricles are important indexes for several cardiovascular diseases. In this paper, a semiautomatic algorithm based on 4D fuzzy connectedness is proposed for segmenting the cardiac ventricles in arbitrarily oriented cardiac cine MR images. The segmentation of the left ventricle was evaluated using 45 short axis images and a visual assessment of right ventricle segmentation was performed.
\end{abstract}

\section{Introduction}

Cardiovascular diseases are the most common causes of death in western nations. Important diagnostic parameters for characterizing heart function are the volumes of the left ventricle (LV) and right ventricle (RV) at the end systolic (ESV) and end diastolic (EDV) time points. Parameters derived from these quantities such as stroke volume $(\mathrm{SV}=\mathrm{EDV}-\mathrm{ESV})$ and ejection fraction $(\mathrm{EF}=\mathrm{SV} / \mathrm{EDF})$ are also commonly reported. The LV and RV can be segmented and quantified in cardiac $\mathrm{CT}$ or $\mathrm{MR}$ images. As these images are $3 \mathrm{D}$ or $3 \mathrm{D}+$ time, manual segmentation is very time consuming and suffers from inter- and intra-observer variability. Therefore, over the last years, several (semi-) automatic approaches have been proposed for segmenting the LV and RV. A particular focus has been on the segmentation of the LV, which is larger and stronger than the RV, and also more easily segmented due to its regular conic shape. Strongly model-based active shape approaches have therefore been devised for the LV, see for example the MICCAI 2009 LV Segmentation Challenge ${ }^{1}$. In contrast, the RV is more difficult to segment due to a more complex anatomy and weaker image contrast. Moreover, model-based approaches are often optimized for one image orientation, and they experience difficulties for patients with severe heart diseases and with non-standard anatomy, e.g., in congenital heart disease. In this work, a semi-automatic 4D fuzzy connectedness algorithm is proposed for a $4 \mathrm{D}$ segmentation of the LV and RV. No specific shape information is assumed and the method is therefore not restricted by the image view or pathologic anatomies.

\footnotetext{
${ }^{1}$ http://smial.sri.utoronto.ca/LV_Challenge/Home.html
} 
There are two main contributions of this paper: First, the 3D fuzzy connectedness algorithm in [1] has been extended to 4D. Second, an image processing pipeline based on the 4D fuzzy connectedness for segmenting the LV and RV from cardiac cine MRI images has been developed. Fuzzy connectedness has previously been proposed for heart ventricle segmentation $[2,3,4]$, but only for the LV and 3D.

\section{Materials and Methods}

\section{$2.14 \mathrm{D}$ fuzzy connectedness}

Udupa and Samarasekera [5] introduced the fuzzy connectedness framework in which the degree of connectedness between two voxels $u$ and $v$ is defined by considering all possible paths between $u$ and $v$. The cost $S[\rho(u, v)]$ of a path $\rho(u, v)$ is defined as the minimal image intensity value along the path. The fuzzy connectedness $C(u, v)$ is now defined by the path with the maximum cost, i.e.,

$$
C(u, v)=\max _{\rho(u, v)} S[\rho(u, v)]
$$

Seeing the image as a height map, the fuzzy connectedness is defined by the path between $u$ and $v$ along which the lowest height is as large as possible, and the connectedness is this minimum height. Tizon and Smedby [1] proposed a fast 3D implementation of the fuzzy connectedness technique using a forwardback scan. In this work, this algorithm was extended to handle up to $6 \mathrm{D}$ images and it was implemented in $\mathrm{C}++$ in the free software platform MeVisLab (www.mevislab.de). The forward scan mask for 4D fuzzy connectedness is shown in Fig. 1. The fuzzy connectedness framework can be used for image segmentation by calculating the degree of connectivity for each voxel in the image to a set of seed voxels of different classes or labels. Each voxel is classified according to which seed point it has the largest connectivity.

\subsection{Segmentation of the left and right ventricle}

The classic segmentation approach using the fuzzy connectedness is to calculate the connectedness map and apply a threshold to it. The goal here is provide

Fig. 1. Neighbourhood masks at timepoint $\mathrm{t}-1$ (A) and $\mathrm{t}(\mathrm{B})$.
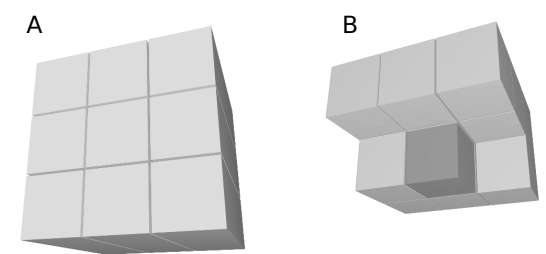


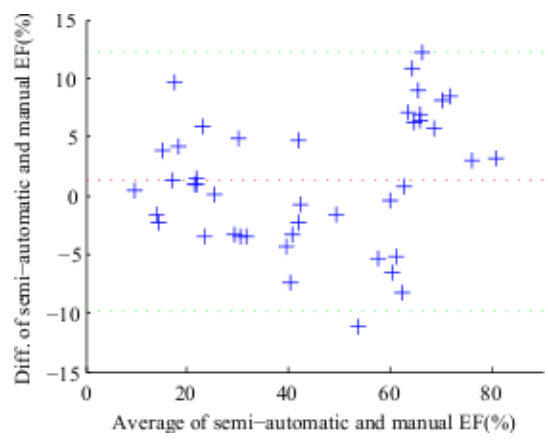

Fig. 2. Bland altman plot comparing the manual and semi-automatic measurement of the ejection fraction.

a spatio-temporal segmentation of the MRI image into three classes: LV, RV and background. To this end, a combination of the classic fuzzy connectedness approach combined with an initial separation of the LV and RV by applying the fuzzy connectedness algorithm to a derivative-of-Gaussian gradient magnitude image is proposed. Manual input is required in form of seed areas in the LV and RV for one timepoint, and the definition of a valve plane to stop the ventricle segmentation from leaking into the aorta. The ventricles are then considered as a $4 \mathrm{D}$ object spatio-temporal objects to be segmented. The fuzzy connectedness algorithm also requires seed markers for the background class, which are straightforwardly derived automatically from the image histogram. In the initial step, the 4D fuzzy connectedness algorithm is applied on a gradient magnitude image, classifying each voxel according to which marker class (LV, RV or background) it has the greatest affinity.

This initial segmentation tends to be under-segmented at the myocardial borders, caused by the application to the a smoothed gradient image. Leakage around the RV can also be observed. Therefore, a second traditional fuzzy connectedness step is applied twice to the original image and for LV and RV separately, i.e., into $\{L V$, background $\}$ and $\{$ RV, background $\}$ respectively. Furthermore, the fuzzy connectedness is only applied to an area of interest which is found by dilating the initial segmentations of the LV and RV by 5 voxels. The thresholds for the connectedness maps are found by an automatic analysis of the grey value distribution inside the initially segmented volumes of the LV and RV, providing the final spatio-temporal segmentation. The papillary muscles will not be included in the segmentation due to their deviating image contrast, but they can be included by calculation the convex hull of the segmentation masks.

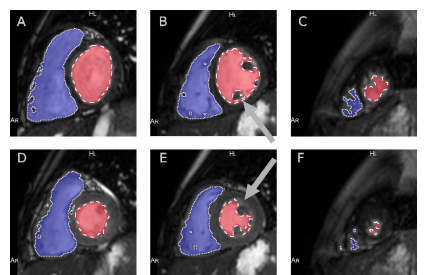

Fig. 3. The images show the delineation of the left(dashed) and right(dotted) ventricular blood pool. In upper row the results in three slices at end diastole, in the lower row three slices at end systole are visualised. The red arrows indicate that the papillary muscles are excluded. 


\subsection{Data}

Cine steady state free precession (SSFP) MR short axis images of 45 subjects from the MICCAI 2009 LV Segmentation Challenge were used for evaluating the LV segmentation. The $4 \mathrm{D}$ images have an in-plane resolution of $256 \times 256$ voxels, $6-12$ slices in the z-dimension, and 20 time points over the heart cycle. A manual delineation of the endocardium at the end-systole and end-diastole phases, including the papillary muscles, is available. In addition, images in short axis and transversal orientations from the Deutsches Herzzentrum Berlin were used for visual assessment of both LV and RV.

\section{Results}

As proposed in the MICCAI Challenge 2009, a Bland-Altman analysis is used to compare the algorithmic results with the manual segmentation, see (Fig. 2) for a Bland-Altman plot for the ejection fraction (EF). The mean difference observed between of the manual and semi-automatic segmentation for the EF is $1.2 \pm 5.6 \%$ and for the volume $13.1 \pm 23.3 \mathrm{ml}$. The best values from the MICCAI Challenge are $1.7 \pm 8.5 \%$ and $22.5 \pm 26.2 \mathrm{ml}$ respectively. Furthermore, the segmentation masks have been compared using the Dice coefficient and the symmetric average perpendicular distance. The mean Dice coefficient is $0.84 \pm 0.07$ and the mean average perpendicular distance (APD) is $2.96 \pm 1.09 \mathrm{~mm}$. As reference, one of the best methods of the MICCAI challenge achieved a mean Dice of $0.89 \pm 0.03$ and a mean APD of $2.29 \pm 0.57 \mathrm{~mm}$. Finally, results of the segmentation of LV and RV in the data from the Deutsches Herzzentrum Berlin are shown in Fig. 3 and Fig. 2.2. Figure 2.2c shows the volumes of the LV (red) and RV (blue) over one heart beat. The interactive segmentation time of one datataset is about 2 to 5 minutes, and the calculation time of the $4 \mathrm{D}$ fuzzy connectedness algorithm is about 5 seconds.

A

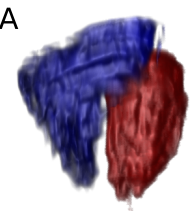

B

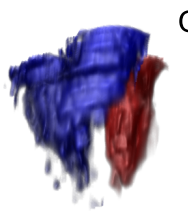

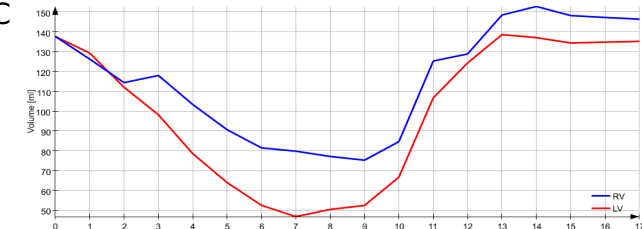

Fig. 4. A and B show the volume rendering of a segmentation result of left and right ventricle corresponding to Fig. 3 at end-diastole(A) and end-systole(B). C visualises the volume curve for each ventricle over one cardiac cycle, where the red curve corresponds to the left and the blue to the right ventricle. 


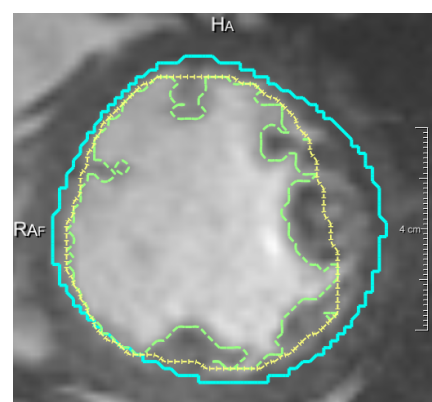

Fig. 5. Dashed (inner): Segmentation result FC. Marked: Convex hull of segmentation result. Solid (outer): Expert contour for myocardial thickness.

\section{Discussion}

The results of the evaluation indicate that the proposed approach provides similar accuracy as a manual segmentation of the left ventricle. Comparing our results with those of 2009 MICCAI Challenge, one can see that the results of the best participants have slightly higher scores regarding the Dice coefficient and the average perpendicular distance, whereas our method is superior in terms of the clinically more relevant EF. Here it should be stressed that the manual segmentations provided by the experts are intended for measurement of myocardial thickness, whereas the proposed method aims to delineate the blood pool (Fig. 5), which may explain this result. Moreover, the proposed algorithm is principally not restricted to specific image orientation, e.g., short axis images, as it works fully 4D. This is in contrast to many model-based algorithms which often work with $2 \mathrm{D}$ contours. In future work the RV segmentation must be evaluated more thoroughly. It was noted in this work that segmentation leakage was a larger problem for the RV than the LV, and that more background seed points must sometimes be set for the fuzzy connectedness algorithm to obtain an accurate result. Further work on placing and tracking the valve plane is also planned.

\section{References}

1. Tizon X, Smedby O. Segmentation with gray-scale connectedness can separate arteries and veins in MRA. J Magn Reson Imaging. 2002;15(4):438-45.

2. Pednekar A, Kurkure U, Muthupillai R, et al. Left ventricular segmentation in MR using hierarchical multi-class multi-feature fuzzy connectedness. Lect Notes Comput Sci. 2004;3216:402-10.

3. Fleureau J, Garreau M, Simon A, et al. Assessment of global cardiac function in MSCT imaging using fuzzy connectedness segmentation. In: Comput Cardiol; 2008. p. $725-8$.

4. Pednekar AS, Muthupillai R, Cheong B, et al. Automatic computation of left ventricular ejection fraction from spatiotemporal information in cine-SSFP cardiac MR images. J Magn Reson Imaging. 2008;28(1):39-50.

5. Udupa JK, Samarasekera S. Fuzzy connectedness and object definition: theory, algorithms, and applications in image segmentation. Graph Models Image Process. 1996;58(3):246-61. 